\title{
Tamper-resistant drugs cannot solve the opioid crisis
}

\author{
Pamela Leece MD MSc, Aaron M. Orkin MD MSc MPH, Meldon Kahan MD
}

See also CMAJ Open article, www.cmajopen.ca/content/3/2/E231

$\mathrm{T}$ amper-resistant formulations, designed to make drugs harder to crush, snort or inject, are being promoted in Canada and the United States as a strategy to prevent opioidrelated harms such as overdose and addiction. In a research article published in CMAJ Open, Gomes and colleagues ${ }^{1}$ examined the effects of tamperresistant opioid formulations on patterns of prescribing near the US-Canada border. Although it is important to understand the effects of such interventions, clinicians should use careful judgment when prescribing any opioid and not be distracted by tamper-resistance as a panacea to reduce opioid-related harms. Regulations requiring tamper resistance will likely do little to address the opioid epidemic and may serve merely to line the pockets of drug companies while diverting policy-makers' attention away from more meaningful and effective interventions.

Misuse and diversion of opioids is a complex problem that requires a comprehensive solution; simply substituting one formulation for another will not work. ${ }^{2}$ Use of narcotics per capita is higher in Canada and the US than in any other country in the world, and drug poisoning is now the primary cause of injury-related death in the US. $^{3}$ In Ontario, oxycodone prescriptions rose $850 \%$ between 1991 and 2007, and opioid-related mortality doubled between 1991 and 2004. ${ }^{4}$ About $80 \%$ of patients entering treatment for opioid abuse were first exposed to opioids through a prescription. ${ }^{5}$ Among individuals whose death was opioid-related, a large proportion (48\%-92\%) had a current prescription for opioids. ${ }^{2,6}$

The term "tampering" refers to the manipulation of prescription opioid tablets, capsules or patches to achieve a more rapid psychoactive effect or "high" through chewing, inhalation, nasal insufflation or injection. Tamper-resistant formulations are designed to prevent tampering in different ways: some release an opioid antagonist such as naloxone to counteract drug activity, and others produce an unpleasant side effect, form a viscous substance when combined with liquid or retain their controlled-release properties.

Chewing opioid pills is a common method of intake among individuals seeking treatment for opioid addiction or reporting nonmedical opioid use, but it is not clear how often chewing is implicated in opioid-related deaths. ${ }^{6,7}$ Tampering with opioid medication for non-oral administration, by injecting, smoking or intranasal snorting, is not a factor in most opioid-related deaths, instances of nonmedical opioid use or admissions to treatment centres. ${ }^{6,7}$

Surveys and observational studies focused solely on oxycodone-specific outcomes have shown some benefits following the introduction of the tamper-resistant formulation. For example, the prevalence of oxycodone use decreased among individuals assessed for drug treatment. ${ }^{8}$ There was also a decrease in deaths involving extended-release oxycodone that were spontaneously reported to the manufacturer. ${ }^{9}$

However, growing epidemiologic evidence shows that the introduction of tamper-resistant formulations has not lowered the rates of opioidrelated deaths at a population level. In Ontario and the US, overall rates of opioid-related deaths have continued to rise since the long-acting formulation of oxycodone (OxyContin) was replaced with a tamper-resistant formulation (in 2012 and 2010, respectively).,10 Rather, there is increasing evidence that individuals shift to other opioids, including uncontrolled formulations such as heroin, when availability of prescription opioids changes. ${ }^{3}$ The rate of death from heroin poisoning in the US accelerated steeply after 2010, when long-acting oxycodone was restricted. ${ }^{3}$ In Ontario, hydromor-
Competing interests: None declared.

This article has been peer reviewed.

Correspondence to: Pamela Leece, pamela. leece@mail.utoronto.ca

CMAJ 2015. DOI:10.1503 /cmaj.150329 
phone and fentanyl prescriptions increased by $79 \%$ and $20 \%$, respectively, between 2009 and 2013 as the provincial government limited oxycodone availability. ${ }^{11}$

There are many reasons why tamper-resistant formulations will not curb the opioid epidemic overall. First, many such formulations simply include naloxone, which can counteract the effects of opioids if administered intravenously, a strategy that only targets abuse related to injection. Because naloxone has limited oral bioavailability, these formulations have little effect on the psychoactive properties when the medication is chewed.

Second, the formulations with physical properties to deter crushing may be used without crushing or by some other means to defeat the tamper-resistance mechanism. ${ }^{8}$

Third, because tamper-resistant formulations are more expensive than generic opioids and may not be covered by private insurers or public formularies, prescription of conventional opioids without tamper-resistant properties will likely continue. In Ontario, the provincial drug plan provides restricted coverage of OxyNEO (a tamper-resistant formulation) and is unlikely to provide wider coverage of other tamper-resistant formulations.

Lastly, opioid prescribing may increase if tamper-resistant formulations are marketed and perceived as being more safe. In the case of OxyContin, aggressive marketing strategies influenced physician prescribing by exaggerating opioid benefits and minimizing risks. ${ }^{2}$ Similar marketing may lead to overprescribing of tamper-resistant formulations, especially if prescribers believe that such formulations reduce prescriber liability or confer safety benefits for their patients. Counterintuitively, companies that make and market opioids will probably see increased profits through overuse of patented tamper-resistant formulations.

It is time to apply a comprehensive, populationlevel strategy to what is essentially an iatrogenic epidemic. Such an approach must include strategies aimed at the prevention of opioid addiction by reducing initial exposure through evidence-based prescribing practices; treatment strategies such as early recognition of aberrant drug-related behaviours through prescription drug-monitoring programs; and better access to effective addiction treatment programs. ${ }^{2}$ Canada's national strategy to tackle harms associated with prescription drugs First Do No Harm - was developed around five streams: prevention, education, treatment, monitoring and surveillance, and enforcement. ${ }^{12}$
Regulations requiring tamper resistance represent an expensive, technical approach that is influenced by pharmaceutical interests and cannot solve the opioid crisis. An evidence-based, multifaceted strategy is needed - one that has real potential to curb opioid-related harms at a population level. Tamper-resistant formulations are a gimmick, and health professionals should not be fooled by them.

\section{References}

1. Gomes T, Paterson JM, Mukati M, et al. Retrospective analysis of trends in dispensing long-acting non-tamper-resistant oxycodone near the Canada-United States border. CMAJ Open 2015. 3:E231-5.

2. Kolodny A, Courtwright DT, Hwang CS, et al. The prescription opioid and heroin crisis: a public health approach to an epidemic of addiction. Annu Rev Public Health 2015;36:559-74.

3. Hedegaard H, Chen LH, Warner M. Drug poisoning deaths involving heroin: United States, 2000-2013 [NCHS data brief no. 190]. Hyattsville (MD): National Center for Health Statistics; 2015.

4. Dhalla IA, Mamdani MM, Sivilotti ML, et al. Prescribing of opioid analgesics and related mortality before and after the introduction of long-acting oxycodone. CMAJ 2009;181:891-6.

5. Cicero TJ, Lynskey M, Todorov A, et al. Co-morbid pain and psychopathology in males and females admitted to treatment for opioid analgesic abuse. Pain 2008;139:127-35.

6. Madadi P, Hildebrandt D, Lauwers AE, et al. Characteristics of opioid-users whose death was related to opioid-toxicity: a population-based study in Ontario, Canada. PLOS ONE 2013;8: E60600.

7. Katz N, Dart RC, Bailey E, et al. Tampering with prescription opioids: nature and extent of the problem, health consequences, and solutions. Am J Drug Alcohol Abuse 2011;37:205-17.

8. Cicero TJ, Ellis MS. Abuse-deterrent formulations and the prescription opioid abuse epidemic in the united states: lessons learned from OxyContin. JAMA Psychiatry 2015;72:424-30.

9. Sessler NE, Downing JM, Kale H, et al. Reductions in reported deaths following the introduction of extended-release oxycodone (OxyContin) with an abuse-deterrent formulation. Pharmacoepidemiol Drug Saf 2014;23:1238-46.

10. Paperny AM. Updated: opioids killing more Ontarians than ever, coroner's numbers show. Global News [Toronto] 2014 Nov 9. Available: http://globalnews.ca/news/1625100/opioids -killing-more-ontarians-than-ever-coroners-numbers-show/ (updated 2014 Nov 18; accessed 2015 June 5).

11. Grant K. Opioid use increases after oxycodone crackdown. Globe and Mail [Toronto]. 2014 July 8. Available: www.theglobeand mail.com/life/health-and-fitness/health/opioid-use-increases-after -oxycodone-crackdown/article19501813 (accessed 2015 June 2).

12. National Advisory Committee on Prescription Drug Misuse. First do no harm: responding to Canada's prescription drug crisis. Ottawa: Canadian Centre on Substance Abuse; 2013.

Affiliations: Dalla Lana School of Public Health (Leece, Orkin), University of Toronto; Women's College Hospital (Kahan), Toronto, Ont.

Contributors: All of the authors contributed substantially to the drafting and revising of the manuscript, gave final approval of the version to be published and agreed to act as guarantors of the work.

Acknowledgements: The authors sincerely appreciate the thoughtful comments and insights from Drs. Rita Shahin (Toronto Public Health), Rosana Pellizzari (Peterborough County-City Health Unit) and Chris Cavacuiti (Department of Family and Community Medicine, University of Toronto) on an earlier draft of this work. 